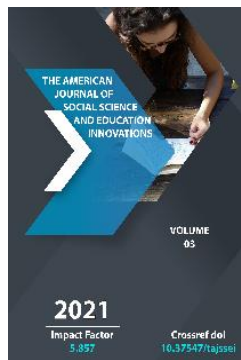

\title{
Problems Of Improving The Effectiveness Of Applied Sociological Research
}

\author{
Farogat Bakhtiyorovna Fayzieva \\ PhD Doctoral Student, Department Of Sociology, Faculty Of Social Sciences, National \\ University Of Uzbekistan
}

\begin{abstract}
Journal Website: http://usajournalshub.c om/index,php/tajssei

Copyright: Original content from this work may be used under the terms of the creative commons attributes 4.0 licence.
\end{abstract}

\section{ABSTRACT}

The article analyzes the problems of improving the effectiveness of applied sociological research. The scientific method that helps to ensure objectivity and consistency in the study of a social problem is considered. The directions, forms and prospects for the development and improvement of the effectiveness of applied sociology from a practical point of view are given.Intelligence research is the simplest type of applied sociological analysis. It solves problems that are very limited in their content, covers, as a rule, small study populations, and is based on a simplified program and concise methodological tools, which in applied sociology is understood as a package of documents specially developed for each study designed to collect primary sociological information, such as: an interview form, a mass or expert questionnaire, a card for recording the results of observation, studying documents; further: sampling projects, mathematical analysis of primary information, etc.A methodology for evaluating the social effectiveness of applied sociological research applied to any written results of scientific activity is proposed.

\section{KEYWORDS}

Efficiency, applied sociology, sociological culture, Imagination sociological, institutional changes, constructs, basic research, forecast of consequences, development, social problems, problem situation, model building. 
Sociology in the modern detailed interpretation is the science of the laws of formation, functioning and development of society, which studies social facts, processes, relations, activities and behavior of individuals and social groups, institutional forms of their organization based on a wide use of empirical data.

Theoretical sociology (also called General sociology) is focused primarily on the study of phenomena and processes on the scale of society as a whole, on the analysis of large social communities, the interaction of social institutions, and ultimately on identifying patterns of functioning and development of society, its main spheres of life. This gives grounds to consider theoretical sociology as a macro-sociology.

Modern sociology is a three-level complex of knowledge (theories, concepts, methods), which is based on the unity and mutual enrichment of the empirical and theoretical, ensuring the integrity of sociological science.

According To M.K.Gorshkov, applied sociology is a system of methodological, methodical and procedural rules and techniques aimed at studying social facts that require verification. [1]

As follows from this definition, applied sociology itself is already divided into three levels (sublevels - if we talk in General about the structure of sociological science): methodological, methodical, and procedural aspects.

The methodological level of applied sociology should be understood as a theoretical or conceptual model (position) based on a certain epistemological paradigm, which underlies the study and interpretation of the subject and research results.

The methodological level is expressed in the implementation of sociological measurement of social phenomena and processes and related methods for collecting and processing empirical information.

\section{RESULTS AND DISCUSSION}

The procedural level is a set of organizational, technical and financial measures necessary for the implementation of the entire technology of sociological research.

As a result of the implementation of a set of rules and techniques included in the levels of applied sociology, it becomes possible to obtain a certain sociological model that serves as the basis for explaining the social phenomenonunder study.[2]

Applied sociological research in its most General form can be defined as a system of logically consistent methodological, methodological and organizational and technical procedures connected by a single goal-to obtain reliable data about the phenomenon or process under study for using this data in the practice of social management.

Applied sociological research includes four successive organizationally Autonomous and at the same time meaningfully interrelated stages:

1) Methodological and methodical preparation of the study;

2) Collection of primary sociological information - non-shared information obtained in the course of a sociological study in various forms (for example, 
respondents ' answers to questionnaire questions, interviews, researcher's notes in observation cards, document analysis, etc.), subject to further generalization;

3) Preparation of the collected information for processing and its computer processing;

4) Mathematical and meaningful analysis of the processed information, preparation of a report, formulation of conclusions and recommendations.[3]

A specific type of sociological research is determined by the nature of the goal set in it, the tasks put forward.

In accordance with them, i.e. depending on the depth of the required analysis of the subject, the scale and complexity of the tasks to be solved, there are three main types of sociological research - intelligence, descriptive and analytical.

Intelligence research is the simplest type of applied sociological analysis. It solves problems that are very limited in their content, covers, as a rule, small study populations, and is based on a simplified program and concise methodological tools, which in applied sociology is understood as a package of documents specially developed for each study designed to collect primary sociological information, such as: an interview form, a mass or expert questionnaire, a card for recording the results of observation, studying documents; further: sampling projects, mathematical analysis of primary information, etc.

This type of research can be used either as a preliminary stage of deep and large-scale research, or to collect "estimated" information about the object of study.
This need arises especially when the subject of sociological research belongs to the category of poorly studied or generally unexplored. In particular, intelligence research is successfully used to obtain additional information about the subject and object, to clarify and correct hypotheses and tasks, methodological tools and boundaries of the surveyed population in in-depth, large-scale descriptive or analytical studies, as well as to identify difficulties that may occur during their implementation.

Performing these auxiliary tasks, intelligence research serves as a provider of operational sociological information. In this case, we can talk about such a variety of it as an Express survey, the purpose of which is to identify people's attitudes to current events and facts (probing public opinion), as well as the effectiveness of various activities. For example, using Express surveys, the audience's satisfaction with the quality of the lecture, the content and form of the lesson is determined. They are often used to assess the progress and results of socio-political campaigns, in particular electoral campaigns.

Descriptive research is a more complex type of sociological analysis. According to its goals and objectives, it involves obtaining empirical information that gives a relatively complete picture of the phenomenon under study, its structural elements. Such research is carried out according to a complete, sufficiently detailed program developed using methodically tested tools. Its reliable methodological equipment makes it possible to group and classify the elements of the object under study according to those parameters that are identified as essential in connection with the problem under study. 
Usually, descriptive research is used when the object of analysis is a relatively large community of people with different characteristics. This may be the team of a large enterprise, where employees of different professions and age groups work, who have different work experience, level of education, marital status, etc., the population of a city, district, region, region, or country. In such situations, the selection of relatively homogeneous groups in the object structure allows us to perform an alternate assessment, comparison and comparison of the characteristics of interest, and to identify the presence or absence of links between them.

Analytical research is the most in-depth type of sociological analysis, which aims not only to describe the structural elements of the phenomenon under study, but also to find out the causes that underlie it and determine its prevalence, stability or variability, and other inherent qualities. Because of this purpose, analytical research is particularly valuable in practice.[4]

If the descriptive study determines the presence (absence) if there are no direct connections between certain parameters of the social phenomenon under study, then the analytical process determines whether the detected connection is causal in nature. For example, in the first case, the researcher may be interested in whether there is a link between employee satisfaction with the content of work performed and its productivity, and in the second - whether satisfaction with the content of work is the only, direct or indirect reason that determines the level of its productivity.

Since the reality of our life is such that it is almost impossible to isolate and study in a "pure form" any one factor affecting it, almost every analytical study considers a set of factors, from which the main and non-main, temporary and stable, explicit and latent (latent), controlled and unmanageable factors are subsequently isolated.

Preparation of an analytical study, including careful development of the program and tools, takes a lot of time. Often, the necessary preliminary information about individual aspects of the object and subject under study, which allow us to choose the best ways to further analyze them in depth, is collected using intelligence or descriptive research.

According to the methods of collecting sociological information, analytical research is complex in nature. Various forms of survey, document analysis, and observation can be used to complement each other. Naturally, this requires the ability to "link" data obtained by different methods and use certain criteria for their interpretation. Thus, analytical research differs significantly from descriptive and intelligence research not only in the content of the preparatory stage and the stage of collecting primary information, but also in the approaches to generalizing and explaining the results.

A social experiment is an independent type of analytical research. Its implementation involves creating an experimental situation by changing (to varying degrees) the usual operating conditions of the object of interest to the researcher. During the experiment, special attention is paid to studying the "behavior" of those factors included in the experimental situation that give this object new properties.

The decision to conduct an experiment should be considered and justified. For example, if we 
are talking about innovations in the field of organizing and stimulating work, about structural changes in certain social institutions, the spread of new forms of mass behavior or group subculture that do not fit into the traditions, in other words, about things that deeply affect personal, group and public interests, we cannot do without preliminary testing on an experimental basis. In such situations, the experiment is not just desirable, but necessary. It allows you to avoid accidents and unforeseen consequences that can lead to mass excesses.

Along with the main criteria (depth, degree of complexity of empirical analysis), by which it is possible to differentiate types of sociological research, other criteria related to the specifics of the method of information collection and the form of research are also used.

Although the public utility of the social Sciences is unlikely to be questioned, the measurement of their public effectiveness is associated with some theoretical problems, without which the construction of an adequate measurement system is hardly possible. The two main problems in this regard are: the difficulty of separating basic social research from applied research, and the categorization of its results in terms of public utility.

It is noted, in particular, that most social studies contain both fundamental and applied elements. Thus, W. Smith points out that in many cases social research is both fundamental in the sense that it sooner or later turns out to be the basis for subsequent research, and applied in the sense that it is focused on solving specific issues or is carried out in the interests of a particular customer. On this basis, the author concludes that the division of aggregated investments in the social Sciences into categories of fundamental and applied research is a "fruitless exercise".[5]

The latter are undertaken with the aim of obtaining knowledge focused on achieving practical goals. At the same time, applied research can be undertaken both to determine possible applications of the results of basic research, and to solve specific, pre-set tasks.

As you can see, applied social research, unlike fundamental research, is aimed at achieving certain practical goals (program development, forecasting consequences, developing support measures) related to solving real-world social problems.

Thus, it seems reasonable to use the concretepractical and target orientation of the former as the main criterion for distinguishing applied social research from fundamental ones.

Currently, there is no clear understanding of the definition of sociological culture.In scientific dictionaries and in many sociological publications, there is a concept of sociological imagination, this concept of "Imagination sociological" is interpreted by CH.Milsom, as a person's self-awareness, the ability to control their behavior through intelligence.[6]

The requirement of today's time is that every personshould have a sociological culture, a sociological idea. Sociological culture is in tune with the concern of a person on the path of finding the purpose of life, not only about himself, but also about the interests of the people around him. Our goals owe their existence to the people around us, their relationships, that is, their mutual existence.

Participants in such surveys are usually referred to as respondents. In a specialized survey, the 
main source of information is competent individuals whose professional activities are closely related to the subject of study or whose theoretical knowledge and life experience allow them to draw authoritative conclusions and give comprehensively balanced assessments. Their participants are called experts, and the surveys themselves are called expert surveys.Sociological observation is a purposeful systematic recording of the properties and characteristics of a phenomenon by a researcher. Among the forms and methods of recording - a form or diary of observation, photo or movie camera, TV and video equipment, etc.

A point study (also called a one-time study) provides information about the state of the object of analysis, the quantitative characteristics of a phenomenon or process at the time of its study. This information is called static, because it reflects, as it were, an instantaneous "slice" of the quantitative parameters of an object, but does not answer the question of trends in its change.

Data reflecting changes in the object can only be obtained as a result of repeated studies based on the same program and tools and conducted sequentially at certain time intervals. In fact, we are talking about a means of comparative sociological analysis, which is aimed at identifying the dynamics of the object under study.[7]

Repeated collection of information can take place in two or three stages or more. The duration of the interval between the initial and repeated stages is very different, because social processes have different dynamics and cyclical changes. Often, it is the object's properties that suggest the time intervals for repeated studies. For example, if we study the trend of implementing the professional attitudes of secondary school graduates and they were interviewed for the first time before the final exams, then it is obvious that the next time for a second survey is no earlier than September - October, when those who have not entered the University are trying to get a job.

Panel research is a special type of repeated research. If, for example, a regular repeated survey is used to study a group of respondents, regardless of how their composition has changed during the time between the initial and repeated stages, then the panel study involves repeatedly contacting the same persons at specified time intervals. Therefore, it is advisable to observe intervals that allow maintaining the maximum stability of the population in terms of its size and composition.

Thus psychologically, any phenomenon can be studied at the level of intelligence, descriptive or analytical sociological research, which has a point or repeated (panel) nature and uses various methods of collecting primary information. In turn, for any type of sociological research, there is no "ban" on the analysis of certain phenomena and processes: each, as already noted, is able to "embed" itself in the subject of study in its own way and provide certain sociological information about it.

When we have different dreams and goals in our youth, as we get older, we do not notice how they change depending on our environment, our society, so that sometimes our old dreams seem frivolous, even ridiculous.

Social, spiritual and cultural changes in our life are closely linked like a chain on the basis of the social environment and being, the behavior 
and actions of people in social reality, and their relationships.

Pointing out the importance of the informational function of social research in terms of making policy decisions, W. Smith recognizes that measuring their specific economic effect is fraught with a number of serious difficulties. This, in particular, requires accurate information about the utility function of economic participants and the production technologies they use.

The problem is even more complicated when it comes to intangible benefits, such as improving the environmental situation, getting out of a recession, or reducing crime. In General, the author acknowledges that the effects of applied social research focused on specific policy issues cannot be adequately identified by existing econometric methods.[8]

Thus, both approaches analyzed above, in our opinion, cannot be used as the basis for a General system of categorization of social effects in applied social research. Their main drawback is related to the use of the concept of economic efficiency, which does not allow us to adequately measure the non-monetary effects of social Sciences related to the public goods they produce.

B. suggests a different approach. Rattan. He believes that the main effects of social research are determined by their ability to stimulate institutional change.

At the same time, institutions are understood very broadly by Rattan: they are not only "rules of behavior that regulate patterns of relationships and actions", but also "decisionmaking centers", which include public authorities, businesses and families.[9]
Institutional changes are understood as the destruction of old institutions and the creation of new ones, as well as changes in their functioning. These transformations can create new sources of revenue and reduce the cost of conflict resolution.

It is the progress in the social Sciences that has allowed, according to Rattan, to move from slow improvements through trial and error, typical of previous eras, to the thoughtful use of socio-scientific knowledge to design and induce institutional innovations.

As rattan points out, His approach is fundamentally different from social evolutionism, which assumes a slow process of collective learning. In his opinion, there is no guarantee that the resulting institutions can provide effective ways of cultural, social or economic development. Therefore, the task of social Sciences is not only to analyze the ongoing social and economic processes, but also to develop methods for effective intervention in these processes.[10]

The approach proposed by Rattan, which links the social effect of socio-scientific knowledge with its active participation in institutional changes, makes it possible to conceptualize the results of applied social research in terms of setting and solving specific social problems. In this case, it is also possible to take into account the inherent nature of these results of public goods.

As a basis for the formulation of evaluation categories of the social effect of applied social research, it seems useful to put the concept of social construction developed by G. V. Osipov, which is understood as scientific activity aimed at creating desirable social realities and preventing negative developments. 
The social construction algorithm includes six stages::

1) Setting a social goal;

2) Identification of a social problem;

3) Analysis of problem situation factors;

4) Theoretical solution of the problem;

5) Building a problem solving model and

6) Practical implementation of the solution (recommendations).[11]

As you can see, the algorithm is a system in which each next step follows logically from the previous one and is practically impossible without it. The implementation of the entire algorithm can be considered as a complete solution to a social problem and can be accepted as the highest criterion for the social significance of applied social research. It seems appropriate for the evaluation of applied social research to combine the first stage with the second in the framework of setting a social problem, which involves taking into account value considerations in its justification.

In this case, the scale of criteria for evaluating publications in the field of applied social Sciences may look like this.

Assess applied work in the field of social Sciences:

1) Poses a social problem;

2) Analyzes the factors of the problem situation;

3) Offers a theoretical solution to the problem;

4) Contains empirical confirmation of the theoretical solution by conducting experiments, field studies, surveys, surveys, computer simulations, etc.;

5) Offers specific recommendations for solving the problem.

\section{CONCLUSION}

Thus, the lowest rating given to the work, limited to the implementation of the first phase of the algorithm for solving social problems, and the higher - implemented the algorithm completely. The proposed methodology for assessing the social effectiveness of applied social research can be applied to any written results of scientific activity. The point of using the above criteria is to standardize the evaluation process, which, on the one hand, allows experts to Express their opinion in a more organized form, and on the other hand, it allows quantifying the evaluation result using a system of coefficients.

\section{REFERENCES}

1. Gorshkov M. K., Sheregi F. E. Applied sociology: methodology and methods. M.: Institute of Sociology PAH, 2011. p11.

2. Dobrenkov V. I. Methodological problems of the formation of theoretical knowledge in sociology //Bulletin of the Moscow University. Sociology. 2014. № 2. C. 3.

3. Gorshkov M. K., Sheregi F. E. Applied sociology: methodology and methods. M.: Institute of Sociology PAH, 2011. p 7 .

4. Mikeshina L. A. Sociology and epistemology: exchange of cognitive experience // Epistemology \& Philosophy of Science. 2015. № 5. p 22.

5. Osipov G. V., Klimovitsky S. V. Evaluation of the public effectiveness of fundamental scientific research // Social and humanitarian knowledge, 2014, № 5, p 54-62. 
6. Лоусон Т., Гэррод А. Социология. А-Я:

Словарь-справочник.пер. с англ. М.:

Фаир-Пресс, 2000. С. 444.

7. Yadov, V. A. Strategy of sociological research. Description, explanation, understanding of social reality: textbook. - M., 2001. p118.

8. SmithV.H. Op. cit., p. 14.

9. Ruttan V. Social science knowledge and induced institutional innovation: An institutional design perspective. Paper prepared for presentation to Conference on Sociological Knowledge for Induced Development, Biblio-theca Alexandriana, May 30 - June 1, 2005, p. 15

10. Ruttan V. Social science knowledge and induced institutional innovation: An institutional design perspective. Paper prepared for presentation to Conference on Sociological Knowledge for Induced Development, Biblio-theca Alexandriana, May 30 - June 1, 2005, p. 15.

11. Osipov G. V. Introduction to sociological science. M., 2010, p 81-82. 